\title{
Research update: The relation between ME/CFS disease burden and research funding in the USA
}

\author{
Arthur A. Mirin ${ }^{\mathrm{a}, *}$, Mary E. Dimmock ${ }^{\mathrm{b}}$ and Leonard A. Jason ${ }^{\mathrm{c}}$ \\ andependent Researcher, Castro Valley, CA, USA \\ ${ }^{\mathrm{b}}$ Independent Researcher, Waterford, CT, USA \\ ${ }^{\mathrm{c}}$ Director, Center for Community Research, DePaul University, Chicago, IL, USA
}

Received 10 November 2019

Accepted 14 January 2020

\begin{abstract}
.
BACKGROUND: Myalgic Encephalomyelitis/Chronic Fatigue Syndrome (ME/CFS) is a debilitating, chronic, multisystem disease that affects an estimated 1 to 2.5 million Americans. It has no widely accepted biomarkers and no FDA-approved treatment. ME/CFS has traditionally been one of the lowest funded diseases by the United States National Institutes of Health (NIH).

OBJECTIVES: We provide here an update to our 2016 article, which estimated the disease burden of ME/CFS in the United States in 2013 and its relation to NIH's 2015 analysis of research funding and disease burden. This update incorporates more recent burden data from 2015 and funding data from 2017.

METHODS: We perform a regression analysis on funding versus disease burden to determine 2017 funding levels that would be commensurate with burden. Burden figures for 2017 are estimated using population-based extrapolations of earlier data. RESULTS: We find the disease burden of ME/CFS is double that of HIV/AIDS and over half that of breast cancer. We also find that ME/CFS is more underfunded with respect to burden than any disease in NIH's analysis of funding and disease burden, with ME/CFS receiving roughly $7 \%$ of that commensurate with disease burden.
\end{abstract}

CONCLUSIONS: To be commensurate with disease burden, NIH funding would need to increase roughly 14-fold.

Keywords: Chronic Fatigue Syndrome, disease burden, burden-commensurate funding

\section{Introduction}

Myalgic Encephalomyelitis/Chronic Fatigue Syndrome (ME/CFS) is a debilitating, complex, chronic disease that affects 1 to 2.5 million Americans of all ages, genders, races, and socioeconomic groups. As the National Academy of Medicine concluded [1],

*Address for correspondence: Arthur A. Mirin, Castro Valley, CA, USA. E-mail: aamirin@comcast.net. patients experience a range of symptoms including sleep abnormalities, cognitive impairment, orthostatic intolerance, pain, flu-like symptoms, substantial impairment in functioning accompanied by fatigue, and the hallmark post-exertional malaise in which even small amounts of physical, mental, or emotional exertion cause an exacerbation of symptoms and further reduction in functioning. Research has demonstrated impairment in the immune, neurological, energy metabolism, and autonomic systems. 
The cause of the disease is unknown, although in many cases, onset follows an infection. There are no FDA-approved treatments.

ME/CFS is more debilitating than many chronic diseases including multiple sclerosis, chronic renal failure, lung cancer, stroke, and type- 2 diabetes [1, 2]. As many as $75 \%$ of patients are unable to work $[3,4]$ and an estimated 25-29\% are homebound or bedbound [1]. In fact, a study by Pendergrast, et al. [5] that examined patients' functional status found that only $16 \%$ of patients were working part or full time. As a result, ME/CFS costs the US economy \$18-24 billion a year in lost productivity and medical costs [1, 6]. Recovery is rare, estimated at 5\%, leaving patients ill for years or decades [7].

In spite of this level of debility, less than one-third of medical schools include ME/CFS in their core curricula $[1,8,9]$, and the clinical guidance used by medical providers in practice includes treatments that are outdated, inappropriate, and potentially harmful $[10,11]$. It is estimated that there are fewer than two dozen nationally recognized ME/CFS specialists in the entire country [12]. Patients struggle to find doctors willing and able to care for them. The US Centers for Disease Control and Prevention (CDC) has referred to ME/CFS as America's Hidden Health Crisis [13]. This crisis in care is accompanied by a remarkably low level of research funding.

In 2016, NIH published a report evaluating the extent to which its funding decisions reflected the burden of disease [14]. This analysis included 68 diseases but did not include ME/CFS. The disease burden was estimated using the Disability Adjusted Life Year (DALY), a measure that estimates the number of years lost due to an illness. The DALY was developed by the World Health Organization [15] and has been used by the NIH to compare its allocation of funding across selected diseases. The DALY folds prevalence, morbidity (the extent of disability), and mortality (the rate and prematureness of death) into a single measure that represents the sum of the years lost due to disability (YLD) and the years lost due to death (YLL); it can therefore be used to compare impacts of primarily disabling diseases with those of primarily deadly diseases.

Rockey and Wolinetz [16] noted that in addition to disease burden, NIH's funding decisions also consider scientific merit, scientific opportunity, portfolio balance, and budgetary considerations. One would expect that disease burden would weigh heavily amongst these factors to ensure that the most burdensome diseases received an equitable share of funding.
Yet, as pointed out by Carolyn Johnson, the NIH funding for AIDS in 2015 was \$3.1B while the funding for a deadly lung disease with six times the disease burden (chronic obstructive pulmonary disease, (COPD)) was 26-fold less at $\$ 118 \mathrm{M}$ [17]. Noting the association between COPD and smoking, Claiborne Johnston, dean of the Dell Medical School in Austin, Texas, stated "we tend to underfund things where we blame the victim." [17]. Unfortunately, ME/CFS has been similarly stigmatized. As a result, between 1995 and 2014, NIH funding for ME/CFS averaged just $\$ 5 \mathrm{M}$ a year, placing it near the bottom of diseases funded [18].

In our earlier journal article [19], we estimated the ME/CFS DALY as a measure of the burden of disease in the United States for 2013. We then compared that and NIH's research funding for ME/CFS to the data used by NIH in its 2015 analysis of disease burden and funding [16]. To calculate the ME/CFS DALY, the 2016 paper used the most commonly accepted adult prevalence of $0.42 \%$ from the 1999 study of Jason, et al. [20], and added to that a pediatric (ages 13-17) prevalence of $0.181 \%$ from the 2006 study of Jordan, et al. [21]. The calculation of morbidity involves computing a disability weight, a number ranging from 0 (not disabling) to 1 (fully disabling). We used a disability weight value of 0.46 , based on work done in Australia [22] and Denmark [2].

Our mortality estimate was based on cohorts of Bateman, et al. [23], Jason, et al. [24] and McManimen, et al. [25], which demonstrated increased mortality due to higher incidence of cancer, heart disease and suicide. The resulting ME/CFS DALY of 0.714 million (for the year 2013) consisted of a morbidity component of 0.488 million and a mortality component of 0.226 million. As noted in the 2016 paper, there are significant limitations with the evidence base for ME/CFS, including particularly the lack of robust studies of prevalence and the paucity of research into the mortality and morbidity of ME/CFS. Thus, the resulting DALY estimate should be considered preliminary but nonetheless instructive.

This new study reported herein is an update to our 2016 journal article [19]. We utilize 2015 disease burden figures and 2017 funding information provided by NIH.

\section{Burden comparison across diseases}

We compare the disease burden of ME/CFS in the United States to the other diseases in NIH's analysis. 


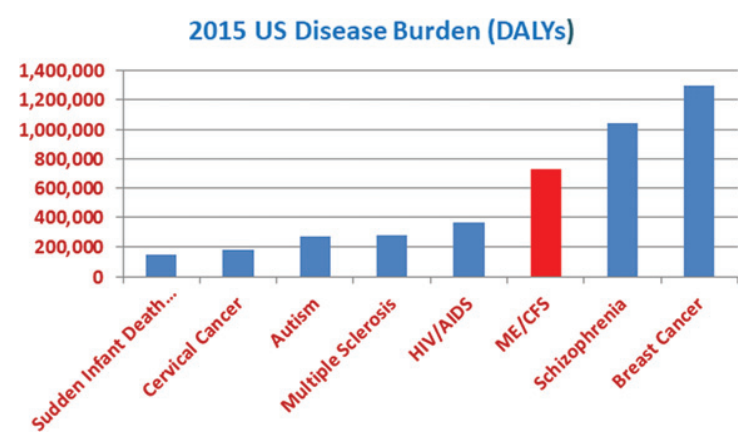

Fig. 1. The US disease burden of ME/CFS relative to that of wellknown, impactful diseases.

We use 2015 disease burden data provided by NIH [26] for diseases other than ME/CFS. The estimate for ME/CFS disease burden provided by Dimmock, et al. [19] is scaled to reflect the growth in the US population between 2013 and 2015. This is a reasonable approximation since in that timeframe, no $\mathrm{ME} / \mathrm{CFS}$ treatments were introduced that might have had a noticeable effect on disease burden.

Figure 1 shows the disease burden, measured in DALY, of various well-known, serious diseases. The disease burden of ME/CFS is seen to be double that of HIV/AIDS and over half that of breast cancer. This illustrates that ME/CFS impacts Americans to an extent comparable to that of other major diseases.

\section{Research funding by the National Institutes of Health}

We now examine NIH funding of ME/CFS, as compared to that of other diseases. We use disease burden as a measure, as more highly burdensome diseases would reasonably be expected to be more highly funded, and vice-versa. We use research funding data for the year 2017. Since burden data for 2017 has not yet been provided by NIH, we estimate 2017 disease burden by extrapolating from earlier years based on population growth. Specifically, the ME/CFS burden figure uses the results of Dimmock, et al. [19], and burden figures for other diseases use the 2015 DALY data provided by NIH [26]. For example, between 2013 and 2017 the US population increased by about $2.62 \%$, so we estimate the 2017 ME/CFS DALY by multiplying the 2013 value of $0.714 \mathrm{M}$ by 1.0262 to get $0.733 \mathrm{M}$.

Figure 2 contains a power law regression analysis of NIH funding versus US disease burden. Each blue point represents an NIH-funded disease, with

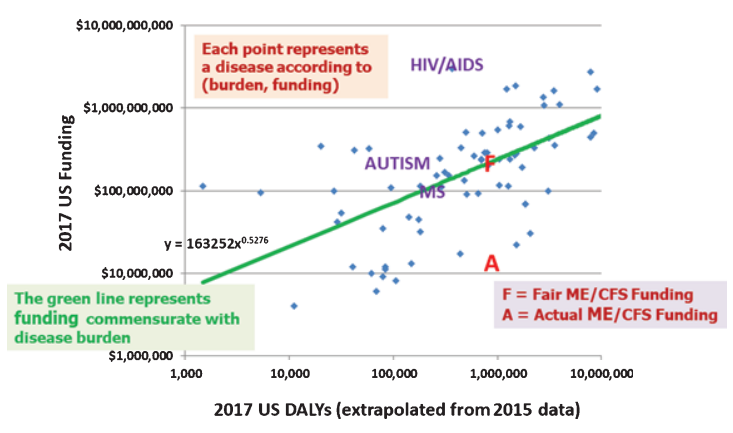

Fig. 2. NIH funding versus US burden of disease.

the horizontal axis measuring burden (in DALYs) and the vertical axis measuring funding. Each axis is plotted on a logarithmic scale in order to represent figures varying over several orders of magnitude. Hence, the spacing between tick marks represents a uniform factor rather than a uniform difference. Furthermore, the resulting power law fit to the data (shown in green) presents itself as a straight line using this double logarithmic scale. Points above the green line may be construed to be overfunded, and those below underfunded. Because of the logarithmic scale, the vertical distance between two points is in effect a difference in logarithms, which is the logarithm of the quotient of the respective values. Hence, two diseases whose points are the same distance below the green line are equally underfunded percentagewise. The red $\mathrm{A}$ and $\mathrm{F}$ represent actual and burdencommensurate ME/CFS funding, respectively. We see that ME/CFS funding needs to be increased roughly 14 -fold to be commensurate with its disease burden.

Figure 3 compares ME/CFS funding to that of similarly burdensome diseases. For this purpose, we consider the nine NIH-provided diseases whose burden is closest to but less than that of ME/CFS, and the nine diseases whose burden is closest to and greater than that of ME/CFS. Note that the vertical scale is logarithmic, so that those diseases closest (in funding) to ME/CFS actually receive almost 10 times as much funding, and many others receive more.

Figures 4 and 5 plot the ratio of actual funding to burden-commensurate funding (the green line in Fig. 2). We see that ME/CFS is the lowest funded of all diseases, at $7.3 \%$ of the burden-commensurate amount. That is, ME/CFS is the most underfunded disease relative to disease burden among the diseases whose burden and funding information are provided by NIH. Figure 4 is limited to underfunded diseases 


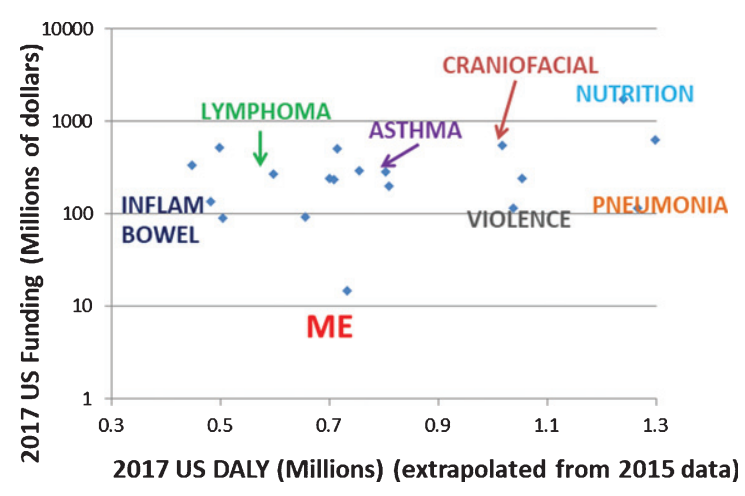

Fig. 3. ME/CFS funding versus that of similarly burdensome diseases.

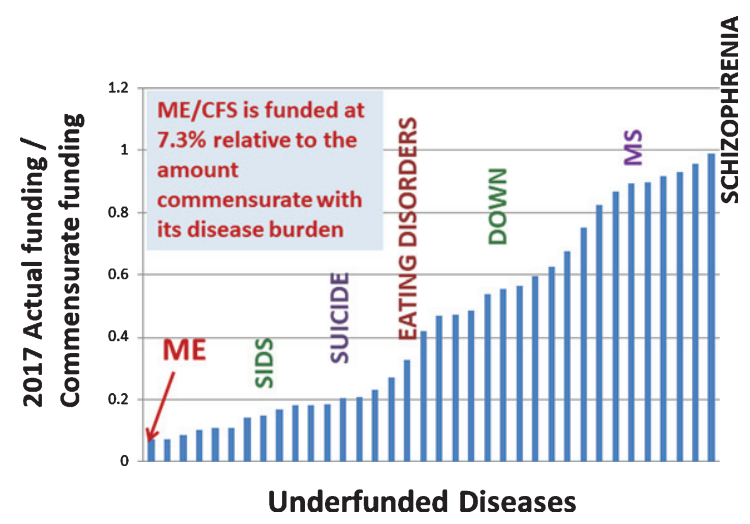

Fig. 4. Ratio of actual to burden-commensurate funding of underfunded diseases.

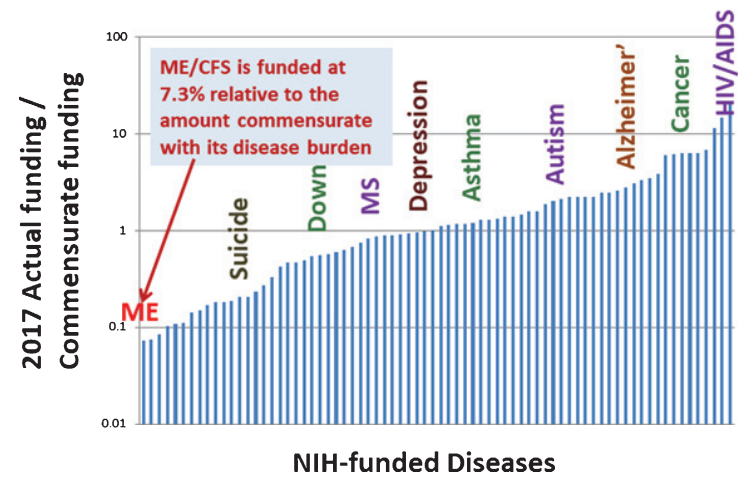

Fig. 5. Ratio of actual to burden-commensurate funding of all diseases for which data is provided.

and enables use of a linear scale. Figure 5 covers all diseases and necessarily must use a logarithmic scale for representation purposes. We note that HIV/AIDS receives roughly 14 times as much funding as its burden-commensurate value.
Table 1

Comparison of actual NIH funding of ME/CFS relative to burden-commensurate funding in 2013 and 2017

\begin{tabular}{lcc}
\hline & 2013 & 2017 \\
\hline Actual ME/CFS funding (\$M) & 5 & 15 \\
Funding commensurate with disease burden (\$M) & 188 & 203 \\
Commensurate minus actual (\$M) & 183 & 188 \\
\hline
\end{tabular}

Table 1 examines ME/CFS funding in years 2013 and 2017. The 2013 burden-commensurate value is from Dimmock, et al. [19]. Despite the approximate tripling of ME/CFS funding during that period (largely due to the enactment of three Collaborative Research Centers and a Data Management Coordinating Center [27]), the shortfall relative to burden-commensurate funding actually increases by $\$ 5$ million.

\section{Limitations}

As noted above, the limitations of the 2016 paper included particularly the lack of robust studies of prevalence and the paucity of research into the mortality and morbidity of ME/CFS. These limitations still exist in this paper, as no additional research has been done to accurately estimate prevalence, mortality, morbidity, or the likely duration in years of ME/CFS.

\section{Conclusions}

We have compared the disease burden of ME/CFS to that of other diseases and shown that ME/CFS is highly impactful, with a 2015 burden double that of HIV/AIDS and over half that of breast cancer. This high burden is due largely to the very low quality-of-life experienced by people with ME/CFS, with many having been very highly debilitated for decades.

We have compared 2017 ME/CFS funding by NIH to that of the other diseases where NIH has provided both funding and burden information. We find that among these diseases, ME/CFS has the lowest 2017 funding relative to disease burden and would need to incur a 14-fold increase for its funding to be commensurate with burden.

This analysis provides an initial estimate of DALY for ME/CFS and hence an initial estimate of the impact of ME/CFS relative to other diseases along with the level of NIH support needed for ME/CFS 
funding to be commensurate with disease burden. The extent of limitations, reported in depth in our 2016 article and summarized herein, underscores the need for a significant ramp-up of research efforts on many fronts, including, for example, searching for biological markers to achieve a better understanding of the disease and its impact and natural history. Also needed are improved tracking and coding practices to better estimate factors such as prevalence, level of disability, and premature mortality.

At the April 2019 NIH-sponsored "Accelerating Research on ME/CFS" Conference, leading researchers echoed the sentiment that the science is indeed ready and that funding must be increased now [28]. While NIH has increased its funding and provided funding for three Collaborative Research Centers, ME/CFS remains the lowest funded disease when compared to disease burden among those where $\mathrm{NIH}$ has provided both disease burden and funding information. Further, as documented by Spotila [29], the establishment of the Collaborative Research Centers corresponded with a decrease in the number of investigator-initiated grants submitted. This is a worrisome trend that is unlikely to be quickly addressed without disease-specific funding opportunities for ME/CFS, including those with set-aside funding to overcome stigma and encourage researchers to enter the field.

In spite of ME/CFS being investigated by NIH and CDC for well over 30 years, little progress has been made on developing diagnostics and treatments, in large part because the research funding provided by the NIH has fallen far short of that which NIH has provided to other diseases of comparable disease burden. To incentivize researchers to enter the field and to accelerate delivery of diagnostics and treatments for people with ME/CFS, NIH will need to substantially ramp up its financial commitment to ME/CFS research. It is time that NIH treat ME/CFS with the urgency that it deserves.

\section{Acknowledgments}

The authors thank Krista Ekberg for reviewing the manuscript and providing helpful comments.

\section{Conflict of interest}

The authors declare no conflict of interest.

\section{References}

[1] NAM - National Academy of Medicine, National Academy of Sciences. Beyond Myalgic Encephalomyelitis/Chronic Fatigue Syndrome: Redefining an Illness; 2015. Available from http://www.nationalacademies.org/hmd/Reports/ 2015/ME-CFS.aspx

[2] Hvidberg MF, Brinth LS, Olesen AV, Petersen KD, Ehlers L. The Health-Related Quality of Life for Patients with Myalgic Encephalomyelitis/Chronic Fatigue Syndrome (ME/CFS). Plos One. 2015;10(7):e0132421. doi: http://dx.doi.org/10.1371/journal.pone.0132421

[3] Lapp C, Unger, ER, Komaroff AL, Nath A. CDC Grand Rounds: Chronic Fatigue Syndrome: Advancing Research and Clinical Education. Centers for Disease Control and Prevention. March 7, 2016. Available from https://www.cdc. gov/grand-rounds/pp/2016/20160216-chronic-fatigue. html. Accessed September 9, 2019.

[4] Unger ER, Lin J-MS, Tian H, Natelson BH, Lange G, Vu D, Blate M, Klimas NG, Balbin EG, Bateman L, Allen A, Lapp CW, Springs W, Kogelnik AM, Phan CC, Danver J, Podell RN, Fitzpatrick T, Peterson DL, Gottschalk CG, Rajeevan MS. Multi-site Clinical Assessment of Myalgic Encephalomyelitis/Chronic Fatigue Syndrome (MCAM): Design and Implementation of a Prospective/Retrospective Rolling Cohort Study. Am J Epidemiol. 2017;185(8):617-26. Available from https://www. ncbi.nlm.nih.gov/pmc/articles/PMC5565838/

[5] Pendergrast T, Brown A, Sunnquist M, Jantke R, Newton JL, Strand EB, Jason LA. Housebound versus Nonhousebound Patients with Myalgic Encephalomyelitis and Chronic Fatigue Syndrome. Chronic Illness. 2016;12(4):292-307. doi:10.1177/1742395316644770. Available from https:// www.ncbi.nlm.nih.gov/pmc/articles/PMC5464362/

[6] Jason L, Benton MC, Valentine L, Johnson A, TorresHarding S. The Economic impact of ME/CFS: Individual and societal costs. Dynamic Medicine. 2008;7(6). Available from https://dynamic-med.biomedcentral.com/articles/10. 1186/1476-5918-7-6

[7] Cairns R, Hotopf M. A systematic review describing the prognosis of chronic fatigue syndrome. Occupational Medicine. 2005;55(1):20-31. Available from https:// www.ncbi.nlm.nih.gov/pubmed/15699087

[8] Jason LA, Paavola E, Porter N, Morello ML. Frequency and content analysis of chronic fatigue syndrome in medical text books. Australian Journal of Primary Health. 2010;16(2):174-8. Available from https://www.ncbi.nlm. nih.gov/pmc/articles/PMC3691015/

[9] Peterson TM, Peterson TW, Emerson S, Regalbuto E, Evans MA, Jason LA. Coverage of CFS within U.S. medical schools. Universal Journal of Public Health. 2013;1(4):1779. Available from http://www.hrpub.org/journals/article_ info.php?aid=821

[10] Doucleff M. For People With Chronic Fatigue Syndrome, More Exercise Isn't Better. National Public Radio, Inc., October 2, 2017. Available from https://www.npr.org/ sections/health-shots/2017/10/02/554369327/for-peoplewith-chronic-fatigue-syndrome-more-exercise-isnt-better

[11] Geraghty K, Jason L, Sunnquist M, Tuller D, Glease C, Adeniji C. The 'cognitive behavioural model' of chronic fatigue syndrome: Critique of a flawed model. Health Psychology Open. 2019;6(1). doi: https://doi.org/10.1177/205 5102919838907 
[12] Griffin S. What's Next for the ME/CFS Clinician Coalition? Bateman Horne Center; 2019. Available from https://batemanhornecenter.org/whats-next-cfs-cliniciancoalition/

[13] CDC. America's Hidden Health Crisis: Hope for Those Who Suffer from ME/CFS; 2017. Available from https://web.archive.org/web/20170616205301/https:/blogs. cdc.gov/publichealthmatters/2017/05/me-cfs/

[14] NIH - National Institutes of Health. Report on NIH Funding vs. Global Burden of Disease; 2016. Available from https://report.nih.gov/info_disease_burden.aspx. Accessed November 11, 2016.

[15] WHO - World Health Organization. About the Global Burden of Disease (GBD) project; undated. Available from http://www.who.int/healthinfo/global_burden_disease/ about/en/. Accessed October 1, 2016.

[16] Rockey S, Wolinetz C. Burden of Disease and NIH Funding Priorities. Rock Talk. National Institutes of Health. June 19, 2015. Available from https://nexus.od.nih.gov/all/ 2015/06/19/burden-of-disease-and-nih-funding-priorities/

[17] Johnson CY. Why the diseases that cause the most harm don't always get the most research money. Washington Post. July 17, 2015. Available from http://www. washingtonpost.com/blogs/wonkblog/wp/2015/07/17/whythe-diseases-that-cause-the-most-harm-dont-always-getthe-most-research-money/

[18] NIH - National Institutes of Health. Estimates of Funding for Various Research, Condition, and Disease Categories (RCDC); 2019. Available from https://report.nih.gov/ categorical_spending.aspx

[19] Dimmock ME, Mirin AA, Jason LA. Estimating the disease burden of ME/CFS in the United States and its relation to research funding. Journal of Medicine and Therapeutics. 2016; 1. Available from http://www.oatext.com/pdf/JMT1-102.pdf

[20] Jason LA, Richman J, Rademaker AW, Jordan KM, Plioplys AV, Taylor RR, McCready W, Huang C-F, Plioplys S. A Community-Based Study of Chronic Fatigue Syndrome. Archives of Internal Medicine. 1999;159(18):2129-37. doi: http://dx.doi.org/10.1001/archinte.159.18.2129

[21] Jordan KM, Huang CF, Jason LA, Richman J, Mears CJ, McCready W, Katz BZ, Ayers, PM, Rademaker A, Taylor KK. Prevalence of Pediatric Chronic Fatigue Syndrome in a Community-Based Sample. Journal of Chronic Fatigue Syndrome. 2006;13(2-3):75-78. doi: http://www.tandfonline.com/doi/abs/10.1300/J092v13n02_ 04
[22] Begg S, Vos T, Barker B, Stevenson C, Stanley L, Lopez AD. The burden of disease and injury in Australia 2003. Australian Institute of Health and Welfare. Cat. No. PHE 82; 2007. Available from http://www.aihw.gov.au/publicationdetail/?id=6442467990

[23] Bateman L, Darakjy S, Klimas N, Peterson D, Levine SM, Allen A, Carlson SA, Balbin E, Gottschalk G, March D. Chronic fatigue syndrome and co-morbid and consequent conditions: evidence from a multi-site clinical epidemiology study. Fatigue: Biomedicine, Health and Behavior 2015;3(1):1-15. doi: http://www.tandfonline.com/doi/abs/ 10.1080/21641846.2014.978109

[24] Jason LA, Torres-Harding S, Njoku MG. The Face of CFS in the U.S. The CFIDS Chronicle. January 2006. Page 3. Available from https://www.researchgate.net/profile/Leonard Jason/publication/236995875_\%20The_Face_of_CFS_in_ the_U.S/links/00b7d51acf6823bccb000000.pdf

[25] McManimen SL, Devendorf AR, Brown AA, Moore $\mathrm{BC}$, Moore JH, Jason LA. Mortality in patients with myalgic encephalomyelitis and chronic fatigue syndrome. Fatigue: Biomedicine, Health and Behavior. 2016;4(4). October 12, 2016. doi: http://dx.doi.org/10.1080/21641846. 2016.1236588

[26] NIH - National Institutes of Health. Report on NIH Funding vs. Global Burden of Disease; 2017. Available from https://report.nih.gov/info_disease_burden.aspx. Accessed June 26, 2018.

[27] NIH Announces Centers for Myalgic Encephalomyelitis/ Chronic Fatigue Syndrome (ME/CFS) Research; 2017. Available from https://www.nih.gov/news-events/newsreleases/nih-announces-centers-myalgic-encephalomyelit is-chronic-fatigue-syndrome-research

[28] Tucker, M. 'Milestone' Meeting Highlights NIH Efforts to Combat ME/CFS; 2019. Available from https://www. medscape.com/viewarticle/911906?src=wnl_edit_tpal\&uac $=265282$ FG\&impID $=1939789 \&$ faf=1\#vp_1

[29] Spotila J. The NIH is thwarting research on a poorly understood yet serious condition; 2019. Available from https://www.statnews.com/2019/01/10/nih-obstaclesthwart-myalgic-encephalomyelitis-research/ 